\title{
EDITORIAL
}

\section{Do we have a niche market?}

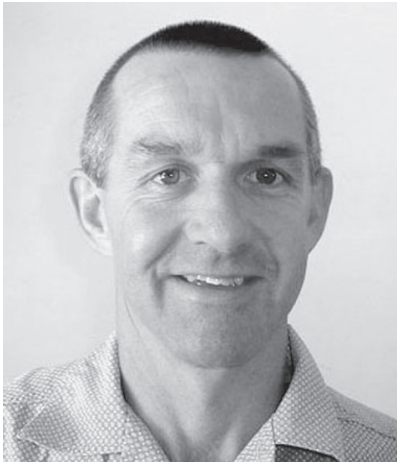

My attention was drawn to a paper which examined the characteristics of successful journals. ${ }^{1}$ In the study, editors of successful journals were interviewed and questioned about their views on factors which had contributed to the success of their respective journals. Some of the main points arising from this study are listed below:

- Active recruitment of high-impact article - editors personally approached leading investigators in different research areas in their quest to attract high-quality papers to their journals.

- Improving services to authors - editors attempted to attract authors by speeding up the turnaround times and fast-tracking publications which had a particularly high impact.

- Finding niche areas - editors attempted to make their journal the definitive journal for that particular specialist area.

- Promoting the journals through media and other forms of communication - this characteristic was supported by a study which showed that research papers from the New England Journal of Medicine, which were also published and popularised in the New York Times, received more scientific citations than articles which did not receive the same treatment. ${ }^{3}$ In other words, scientific impact was improved if the work was communicated beyond the regular scientific/ clinical community.

What can the South African Journal of Sports Medicine learn from this study? While we are in a completely different league to the heavyweight journals surveyed in the study, ${ }^{1}$ there are many practical points from the results of this study which can be considered and implemented. Firstly, we need to seek and canvass for articles from top researchers and clinicians rather than waiting for articles to be submitted. At the 2008 South African Sports Medicine Association conference in Pretoria some clinicians presented fascinating case studies. Most of these presentations have not been converted into publications. The possible reason for this is that the clinicians may not have had much research experience, and feel intimidated about writing the paper. This is something which can be addressed. We will commit to facilitate this process in an attempt to publish cuttingedge applied clinical and performance-related case studies. We already have structures in place to nurture young researchers who are attempting to publish their work and we will attempt to extend this to clinicians who have interesting case studies to report.

Secondly, we need to improve our communication with our readers, informing them about the upcoming editions and interesting articles they can look forward to reading. Soon a mechanism will be in place for better communication between SASMA members, so we will be able to piggyback onto this communication structure to inform members about the journal.

Thirdly, we will also attempt to make the reviewing process as seamless as possible and completed within four weeks. We will attempt to do this without compromising the standard of the review, as a high standard of review is essential for the quality of papers which are published. We have also decided to include a continuing medical education section in the journal, which will serve as an adjunct to the research papers. There are also possibilities about linking this continuing medical education to CPD points.

Fourthly, we need to develop and define our niche area. Our journal needs to become the international mouthpiece for sports medicine and the allied disciplines in South Africa. I think we can assume we will have achieved this goal when the South African Journal of Sports Medicine is the first port-of-call for anyone (local or international) seeking information relevant to sports medicine in South Africa. The fact that the journal has a regional niche should not be seen as an inferior option and therefore a dumping ground for a paper which has an insufficient quality for being accepted for publication in an international journal with a higher impact factor. Rather, our goal should be to publish papers which have not only an international quality, but also a regional interest. Indeed, the ISI classification now includes a regional section catering for journals with these characteristics.

Another goal should be to ensure that a high proportion of the papers published are cited by other studies. It is staggering when one considers that between 1900 and 2005, 38 million papers were published in the clinical medicine and public health field. Half of these papers were not cited at all by subsequent studies in the field. ${ }^{2}$ Our goal should be to ensure that any paper published in the South African Journal of Sports Medicine does not become a statistic and is never cited again.

These are not unrealistic goals. Certainly the points which have been outlined above can be implemented, and there is a very good chance that in so doing the quality of the journal will be increased significantly.

\section{Mike Lambert}

Editor-in-Chief

1. Chew M, Villanueva, Van der Weydon M. Life and times of the impact factor: retrospective analysis of trends for seven medical journals (1994 - 2005) and their editors' views. J R Soc Med 2007; 100: 142-50

2. Garfield E. The history and meaning of the journal impact factor. JAMA 2006; 295 90-3.

3. Phillis DP, Kanter EJ, Bednarczyk B, Tastad PL. Importance of the lay press in the transmission of medical knowledge to the scientific community. N Engl J Med 1991 325: 1180-83 